\title{
The Nightscout system - description of the system and its evaluation in scientific publications
}

System Nightscout - opis systemu i jego ewaluacji w publikacjach naukowych

\author{
Oskar P. Kublin, Mariusz Stępień
}

Department of Propaedeutics of Internal Medicine and Social Pharmacology, Medical University of Lodz, Polska

\begin{abstract}
Nightscout is a non-commercial Do-It-Yourself (DIY) system, developed in 2014 by the parents of children with type 1 diabetes. In Poland it has been available and supported since 2016. The Nightscout system is not an officially registered solution and patients use it at their own responsibility. The idea of the project is to give authorized users online access to data on continuous glucose monitoring in interstitial fluid. Data are stored on external servers. Glucose data can be read on any number of devices with access to the Internet.

This article describes the Nightscout system - required components, operating costs, and other usage options. It also presents the current evaluation of the Nightscout system in scientific publications.
\end{abstract}

Key words:

type 1 diabetes mellitus; DIY - do it yourself; CGM - continuous glucose monitoring; Nightscout system. 


\section{Introduction}

Systems for continuous glucose monitoring (CGM) in interstitial fluid are becoming increasingly popular in the management of diabetes mellitus. These systems allow patients with diabetes easier and continuous access to real-time data on their glucose levels. Clinical trials revealed the positive effect of CGM systems on the course of diabetes treatment. Lower values of glycated haemoglobin, lower number and duration of hypoglycaemic episodes, as well as longer normoglycaemia and lower values of standard deviation of mean glucose levels have been reported in users of CGM systems [1, 2]. The benefits of using CGM systems have been reported both in patients treated with multiple daily injections of insulin and in those using personal insulin pumps [3-5]. Moreover, the benefits in older users of CGM systems are greater than in younger subjects [6]. This may be linked with the fact that young people have greater difficulty in making therapeutic decisions. In addition, children without parental control are more vulnerable to pressure from their social environment and are more inclined to ignore therapeutic recommendations. The Nightscout project was initiated to facilitate glucose monitoring by caregivers of people with diabetes mellitus [7].

Nightscout is a non-commercial do-it-yourself (DIY) system. It was developed in 2014 by the parents of children with type 1 diabetes. In Poland it has been available and supported since 2016 [8, 9]. The Nightscout system is not an officially registered solution, and patients use it at their own responsibility. The Nightscout system operates on open-source software, which means it can be continuously updated. The idea of the project is to give authorised users online access to data on continuous monitoring of glucose in interstitial fluid. The system is also called CGM in the Cloud. Data are stored on external servers: Github, Azure, Mlab (modelling laboratory), and Heroku. Glucose data stored on the servers can be read on any number of devices with access to the Internet (smartwatch, smartphone, laptop) both by the user him/herself and by his/her caregivers. Users of the Nightscout system have to have a device for the monitoring of glucose concentration in the interstitial fluid.

\section{How does it work?}

Currently, the Nightscout system is compatible with the Abbott Freestyle Libre system (flash glucose monitoring - FGM) and CGM systems. When using the Freestyle Libre system, an additional device is required for reading data from the sensor via NFC (Blucon or MiaoMiao), and their transmission via Bluetooth to the phone. In Poland, available CGM devices compatible with Nightscout are offered by Medtronic (MiniMed 640G, VEO, series 7XX and 5XX and Guardian Connect), Dexcom (G4 Platinum, G5), and Roche (Eversense $X \mathrm{~L}$ ). Medtronic devices 640G, VEO, 5XX, and 7XX require the connection of an additional communication module using an OTG (on-the-go) USB cable. In the case of using the uploader for a $640 \mathrm{G}$ pump, in addition to the glucose value in the interstitial fluid, the user may receive information about active insulin, the basal insulin dose, the amount of insulin in the reservoir, and the glucose trend. Users of the Guardian Connect device have to have an account in the CareLink corporate web service, which should be linked to the Nightscout website. In the Dexcom G4 Platinum system, the receiver connects to the phone via an OTG USB cable or Wi-Fi, but it is necessary to have an additional xBridge (Bluetooth communication) or Parakeet device for signal retransmission from the Dexcom transmitter (in the GSM system).

\section{Evaluation in scientific publications}

The Nightscout project was described for the first time in a scientific publication in 2016 [10]. The authors presented Nightscout as an innovative mobile DIY technology managed by patients. They defined this system as a promising solution for current healthcare needs.

The Nightscout system has been evaluated by researchers from Michigan in an English-language anonymous survey targeted at the "CGM in the Cloud" group $[11,12]$. The purpose of this research was to compare disease and demographic characteristics of people who use and do not use a do-it-yourself (DIY) mobile technology system for diabetes (Nightscout), and to present changes in their health behaviours and outcomes. Based on the responses of 1268 members of the CGM in the Cloud group, they received information about 1157 users of the Nightscout project. Most respondents were children (6-12 years old) with type 1 diabetes (99.4\%) using an insulin pump (85.6\%) and CGM (97.0\%). Their study revealed the positive effect of the Nightscout system. During use of Nightscout, patients presented a significant improvement in $\mathrm{HbA}_{10}$ and quality of life (based on their responses related to situations when diabetes prevented them from performing normal activities, spending time at work or with friends). In addition, they took SMBG less often, gave more boluses, and more often gave insulin without SMBG. Respondents were also asked to specify the number devices on which they display Nightscout data. Most adults viewed their results alone on one device, and among children on average three people viewed data using three devices per viewer.

The analysis of demographic data on the surveyed population in a previous study showed that the vast majority of Nightscout users are children and adolescents, because the project was developed for the remote monitoring of glucose levels in children. However, this solution is also used by adult diabetes patients.

Data stored in the cloud can be accessed by users on electronic devices via the Internet. Moreover, the database in the cloud can be used as a digital version of a paper glucose logbook, which is important for both diabetes patients and the doctors who provide medical care to them. Of note, records are standardised regardless of the system used by the patient for glucose monitoring. In addition to popular electronic devices, 
users of the Nightscout system also declared accessing glucose data via the "Garmin" sports watch with navigation and DIY alarms [12].

\section{Peer support}

It should be borne in mind that the installation of the Nightscout system, its use, adaptation after modifications, or malfunction requires strong involvement and certain IT skills from the user. This problem was emphasised by the respondents who joined the CGM in the Cloud group but finally did not use Nightscout [11]. It is impressive that members of this social network group support each other, and the vast majority of questions related to technical problems are answered by the administrator, which makes the use of the Nightscout system easier, and additionally may improve the therapeutic process. Peer support was the subject of the research conducted among members of the CGM in the Cloud group on Facebook [13]. Patients and caregivers were investigated by semi-structured qualitative interviews. They described peer support as technical, emotional, and medical help, which can go beyond the Internet community. In conclusion, the authors emphasise that peer support can be an instrument of empowerment. Similar conclusions were reached by the authors of a meta-analysis evaluating the use of online social networking services by patients with diabetes mellitus [14].

Interesting conclusions were obtained after analysing 1268 surveys regarding the motivation of patients with diabetes for participation in online forums. The main purpose of joining the CGM group in the cloud was to find out more about the Nightscout project and receive technical support from other members. It should be noted that in many situations, the participants of the study showed much greater trust in a member of the group who was not a medical staff professional, while only $40 \%$ claimed they had received help thanks to forum advice [15].

\section{Costs}

It is worth noting that, despite the free access to the Nightscout system, it is necessary to have a device for downloading data - an uploader (different for individual solutions), the cost of which is usually not relatively high. If the patient uses Freestyle Libre, he/she needs an additional reader to convert the system into a DIY CGM system. When a patient decides to buy an additional reader, he/she receives the CGM system, which is cheaper to maintain compared to the typical CGM system. In addition, the user must create his/her account on the data storage website. Free of charge and paid accounts are available. Continuous access to the Internet is required for data transmission. To view the Nightscout data, users need an electronic device (such as smartphone or smartwatch). There is no limit to the number of devices that can be used for this purpose, but some models are more suitable (they have a larger battery or have less other unnecessary functions).

\section{Other possibilities of using Nightscout}

For most users the Nightscout system is an improvement of the system for continuous glucose monitoring in the interstitial fluid, but for some users it gives a further possibility of using their personal insulin pump in a closed loop system. It should be noted that Nightscout does not require a DIY closed loop, but it can be helpful for the patient by providing a data base. A clinical trial conducted in the Czech Republic revealed that the use of closed-loop DIY Android APS further improves the control of the therapeutic process, even compared to the latest-generation insulin pumps operating with SmartGuard technology [16].

Considering the above data, it is worth emphasising that solutions developed by patients are far ahead of commercial products and should not go unnoticed by medical professionals. There are a limited number of publications on Nightscout and other do-it-yourself solutions, but the results obtained in our article suggest the need for further investigation of DIY projects developed by patients and analysis of such solutions in the context of the efficacy and safety of diabetes treatment.

\section{Conclusions}

The Nightscout system can be regarded as an improvement of the standard continuous glucose monitoring in interstitial fluid. For some users this system can be used as support for activating a closed-loop function in a personal insulin pump. Based on the cited articles, the use of the Nightscout system may improve the efficacy of treatment among patients with type 1 diabetes, and it may have a positive effect on the safety of insulin therapy and quality of life.

\section{Acknowledgments}

The study was financed by the Medical University of Lodz under research project no. 502-03/5-139-03/502-54-230-18. 


\section{References}

1. Bolinder J, Antuna R, Geelhoed-Duijvestijn P, et al. Novel glucosesensing technology and hypoglycaemia in type 1 diabetes: a multicentre, non-masked, randomised controlled trial. Lancet 2016; 388: 2254-2263. doi: 10.1016/S0140-6736(16)31535-5

2. New JP, Ajjan R, Pfeiffer AF, et al. Continuous glucose monitoring in people with diabetes: the randomized controlled Glucose Level Awareness in Diabetes Study (GLADIS). Diabet Med 2015; 32 : 609-617. doi: 10.1111/dme.

3. Šoupal J, Petruzelkova L, Flekac M, et al. Comparison of Different Treatment Modalities for Type 1 Diabetes, Including Sensor-Augmented Insulin Regimens, in 52 Weeks of Follow-Up: A COMISAIR Study. Diabetes Technol Ther 2016; 18: 532-538. doi: 10.1089/ dia.2016.0171

4. Lind M, Polonsky W, Hirsch IB, et al. Continuous Glucose Monitoring vs Conventional Therapy for Glycemic Control in Adults With Type 1 Diabetes Treated With Multiple Daily Insulin Injections: The GOLD Randomized Clinical Trial. JAMA 2017; 317: 379-387. doi:10.1001/jama.2016.19976

5. Foster N, Miller K, Tamborlane W, et al. Continuous Glucose Monitoring in Patients With Type 1 Diabetes Using Insulin Injections. Diabetes Care 2016; 39: 81-82. doi: 10.2337/dc16-0207

6. Wojciechowski P, Ryś P, Lipowska A, et al. Efficacy and safety comparison of continuous glucose monitoring and self-monitoring of blood glucose in type 1 diabetes: systematic review and meta-analysis. Pol Arch Med Wewn 2011; 121: 333-343.

7. Nightscout \#WeAreNotWaiting. The Nightscout Project. site: http:// www.nightscout.info/.
8. Nightscout Polska WeAreNotWaiting. site: http://nightscout.pl/.

9. Nightscout Polska (Poland) Facebook group. https://www.facebook.com/groups/NightscoutPoland (created 24.11.2015).

10. Lee JM, Hirschfeld E, Wedding J. A Patient-Designed Do-It-Yourself Mobile Technology System for Diabetes: Promise and Challenges for a New Era in Medicine. JAMA 2016; 315: 1447-1448. doi: 10.1001/jama.2016.1903

11. Lee JM, Newman MW, Gebremariam A, et al. Real-World Use and Self-Reported Health Outcomes of a Patient-Designed Do-it-Yourself Mobile Technology System for Diabetes: Lessons for Mobile Health. Diabetes Technol Ther 2017; 19: 209-219. doi: 10.1089/dia. 2016.0312

12. CGM in the Cloud. Facebook group. https://www.facebook.com/ groups/cgminthecloud (created 28.04.2014).

13. Gavrila V, Garrity A, Hirschfeld E, et al. Peer Support Through a Diabetes Social Media Community. J Diabetes Sci Technol 2019; 13: 493-497. doi: 10.1177/1932296818818828

14. Toma T, Athanasiou T, Harling $L$, et al. Online social networking services in the management of patients with diabetes mellitus: systematic review and meta-analysis of randomised controlled trials. Diabetes Res Clin Pract 2014; 106: 200-211. doi: 10.1016/j. diabres.2014.06.008

15. White K, Gebremariam A, Lewis D, et al. Motivations for Participation in an Online Social Media Community for Diabetes. J Diabetes Sci Technol 2018; 12: 712-718. doi: 10.1177/1932296817749611

16. Petruzelkova L, Soupal J, Plasova V, et al. Excellent Glycemic Control Maintained by Open-Source Hybrid Closed-Loop Android APS During and After Sustained Physical Activity. Diabetes Technol Ther 2018; 20: 744-750. doi: 10.1089/dia.2018.0214 